JOURNAL DE PHYSIQUE IV

Colloque C8, supplément au Journal de Physique III, Volume 6, décembre 1996

\title{
Non Ergodic Aging in an Orientational Glass
}

\author{
F. Alberici, P. Doussineau and A. Levelut \\ Laboratoire d'Acoustique et Optique de la Matière Condensée, Université P. et M. Curie, Case 78, \\ 75252 Paris cedex 05, France
}

\begin{abstract}
In $\mathrm{K}_{1-\mathrm{x}} \mathrm{Li}_{\mathrm{x}} \mathrm{TaO}_{3}$, small $\mathrm{Li}^{+}$ions are randomly substituted to large $\mathrm{K}^{+}$ions. They take off-centre positions creating therefore electric dipoles and elastic quadrupoles at random sites. This disordered system presents frozen random interactions and frustration. For $x>2.5 \%$, thermal hysteresis signals a structural transition with a first order character towards a ferroelectric low temperature phase. For $x<2.5 \%$, at low temperatures the system is frozen in a disordered phase called orientational glass. Measurements of the dielectric constant $\varepsilon$ and of the elastic constant $\mathrm{c}$ provide a lot of information about the nature of the transition and the relaxation of the $\mathrm{Li}^{+}$ions which has been studied in details and analyzed in the form of Argand diagrams exhibiting a very particular (strophoidal) shape. After zero field cooling from $100 \mathrm{~K}$ to $4.2 \mathrm{~K}$ the time evolution of $\varepsilon$ and $\mathrm{c}$ during $80,000 \mathrm{~s}$ has been followed. The behaviour of the system (amplitude and asymptot) strongly depends on the cooling rate. The fact that the asymptotic limit depends on the initial conditions shows that ergodicity is broken in this system.
\end{abstract}

\section{INTRODUCTION}

Pure potassium tantalate $\mathrm{KTaO}_{3}$ crystallizes in the perovskite structure. When it is cooled down to liquid helium temperature its dielectric constant increases up to 4500 but it never becomes ferroelectric: ferroelectricity is prevented by quantum fluctuations. When small $\mathrm{Li}^{+}$ions are substituted to larger $\mathrm{K}^{+}$ions they take an off-centre position along one of the six [100] directions, thus creating a large electric dipolar moment and also a non-spherical elastic quadrupolar moment. In these $\mathrm{K}_{1-\mathrm{x}} \mathrm{Li}_{\mathrm{x}} \mathrm{TaO}_{3}(\mathrm{KLT})$ crystals, due to the random substitution of $\mathrm{Li}$, the strength of the interaction between two $\mathrm{Li}^{+}$ions is a random variable. These materials have been studied by means of many techniques including NMR, Raman and Brillouin scatterings, dielectric and acoustic spectroscopies, X-ray diffraction, birefringence, second harmonic generation $[1,2]$. Evidences have been obtained that the motion of the $\mathrm{Li}^{+}$ions between their six positions are gradually frozen in at low temperatures and that the nature of the phase which is attained at low temperature depends on the $\mathrm{Li}^{+}$concentration $\mathrm{x}$. At low $\mathrm{x}(<0.025)$ the material enters a disordered phase with no macroscopic polarization called a dipolar glass, which can be viewed as the electric analog of the magnetic spin glass. At larger $\mathrm{x}$ values the material transforms into a disordered tetragonal ferroelectric phase.

In this paper we want to focus on several aspects of the acoustic and dielectric studies of KLT crystals. An electromagnetic wave is coupled to the dipolar moment whereas an elastic wave is coupled to the quadrupolar moment. Therefore these two experimental techniques give complementary information. In a first part we present results showing the different nature of the phase transition for low and high $\mathrm{x}$ values. In the second part we are interested in the relaxation of the $\mathrm{Li}^{+}$ions in the high temperature paraelectric phase for low $x$ revealing a particular distribution of the relaxation times. In a third part we present some new results on kinetics effects in these KLT materials and in particular a spectacular non ergodic behaviour.

\footnotetext{
1 Associated to the Centre National de la Recherche Scientifique
} 


\section{EXPERIMENTS}

In dielectric experiments the complex dielectric constant $\varepsilon^{\prime}-i \varepsilon^{\prime \prime}$ is measured along the [100] direction for frequencies ranging from $10 \mathrm{~Hz}$ to a few $\mathrm{MHz}$. The samples are in shape of parallelepipeds of dimensions around 4 to $6 \mathrm{~mm}$ in each direction with flat and parallel end-faces. One pair of opposite faces has been plated with chromium in order to form a capacitor. The capacity and the conductance were measured with an impedance bridge. They were transformed in the imaginary and real parts of the complex dielectric constant $\varepsilon^{\prime}-i \varepsilon^{\prime \prime}$. In acoustic measurements, longitudinal waves propagating along the [100] direction for frequencies between $30 \mathrm{MHz}$ and $1000 \mathrm{MHz}$ were generated by a $30 \mathrm{MHz}$ resonant $\mathrm{LiNbO}_{3}$ transducer bonded in one face. The attenuation and phase velocity changes were automatically measured in a pulseecho method. They were transformed in the imaginary and real parts of the complex elastic constant $c^{\prime}-i c^{\prime \prime}$. The two kinds of experiments were performed on exactly the same samples. Some of them were grown in our laboratory by S. Ziolkiewicz and some others in Oak Ridge by L. A. Boatner. For all the resuits reported here no external electric field was applied during the recording of the data.

\section{PHASE TRANSITION}

Typical data of the real and imaginary parts of the dielectric constant [3] at several frequencies and of the real and imaginary parts of the elastic constant [4] also at various frequencies are shown as a function of temperature for two KLT samples, one with $x=0.015$ (figure 1) and the other with $x=0.05$ (figure 2). Some data were recorded during heating and some others during cooling. When only one set of data is shown, it means that the two sets of data are undistinguishable on the scale used here.
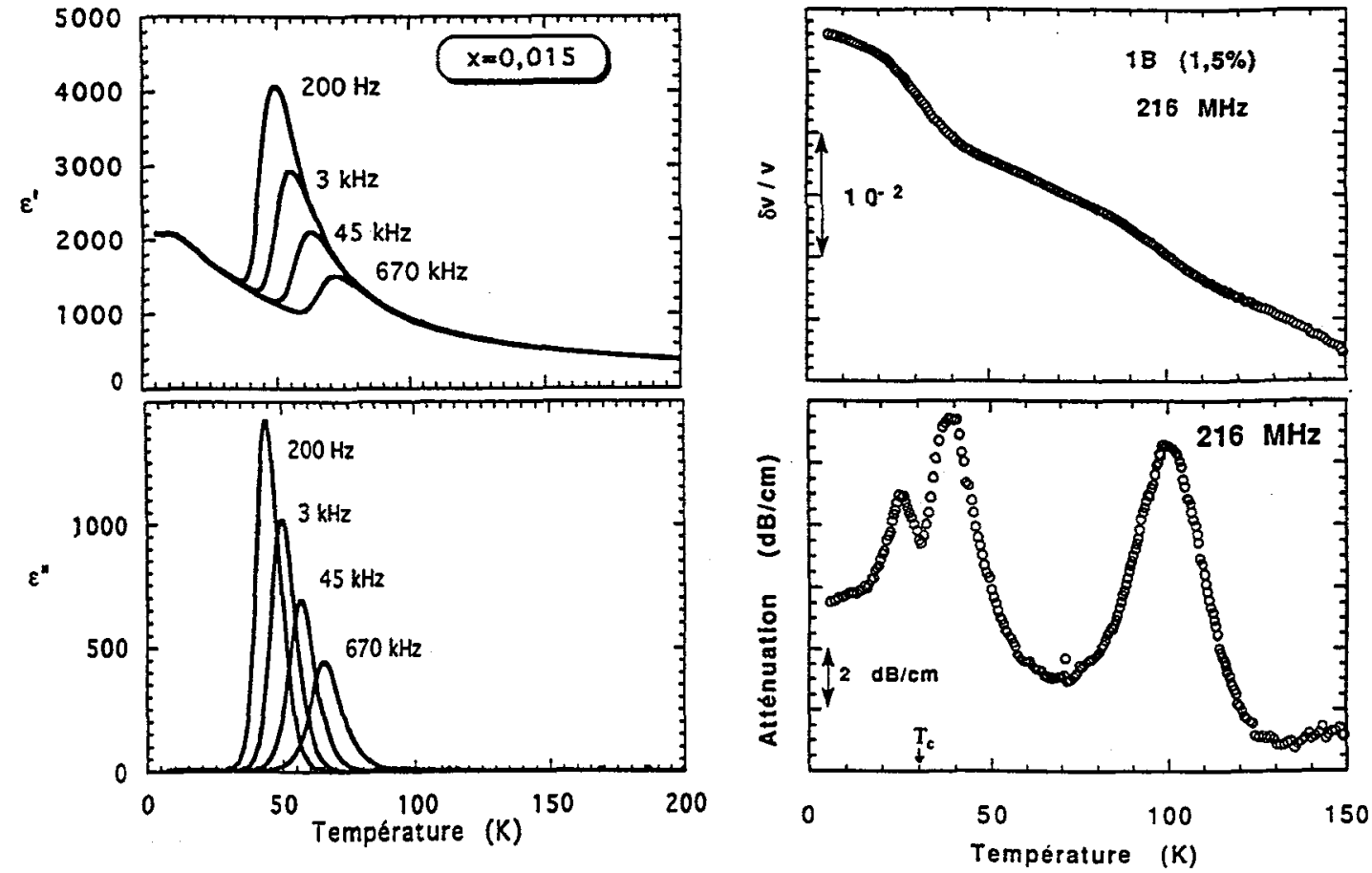

Figure 1 : a) Real part $\varepsilon^{\prime}$ (top) and imaginary part $\varepsilon^{\prime \prime}$ (bottom) of the dielectric constant of a $x=0.015 \mathrm{KLT}$ crystal as a function of temperature for various frequencies. b) Relative variation of the sound velocity (related to the real part $c^{\prime}$ of the elastic constant $\delta c^{\prime} / c_{0}=2\left(\delta v / v_{0}\right)$ (top) and variation of the sound attenuation $\alpha$ (related to the imaginary part $c^{\prime \prime}$ of the elastic constant $\delta c^{\prime \prime} / c_{0}=\alpha v_{0} \ln (10) / 10 \omega_{\text {us }}$ (bottom) as a function of temperature at the frequency $\omega_{\mathrm{us}} / 2 \pi=216 \mathrm{MHz}$ measured in the $x=0.015$ KLT sample. 

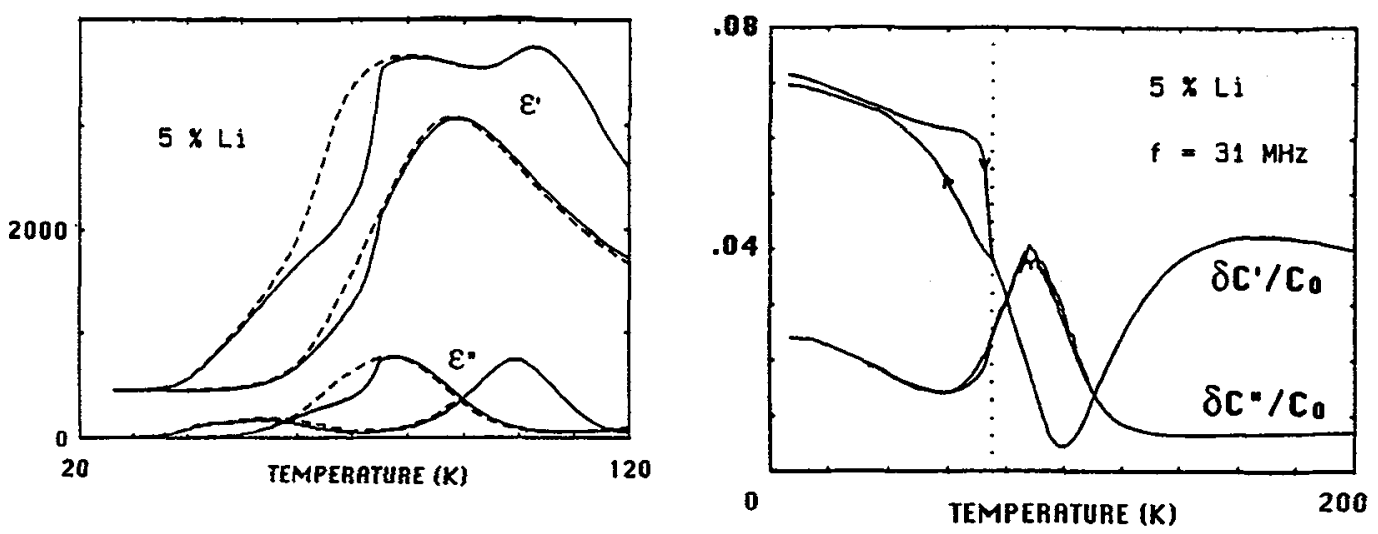

Figure $2:$ a) Real and imaginary parts of the complex dielectric constant of a $x=0.05 \mathrm{KLT}$ crystal as a function of temperature, measured upon cooling (dashed lines) or upon heating (full lines). A vertical division represents 1000 units. The frequencies are $300 \mathrm{~Hz}$ and $300 \mathrm{kHz}$. The $\varepsilon^{\prime}$ curves are above the $\varepsilon^{\prime \prime}$ curves. b) Real and imaginary parts of the complex reiative elastic constant of a $x=0.5 \mathrm{KLT}$ crystal as a function of temperature measured with $31 \mathrm{MHz}$ longitudinal waves.

First we concentrate on the behaviour near the phase transition. It is well observed on the $x=0.05$ sample with a strong similarity of the elastic constant and the dielectric constant. As seen in the figure these two susceptibilities show different values when measured upon heating and upon cooling (hysteresis) both in their real part and in their imaginary part. Moreover the rapid variation which is observed mostly on the real part upon heating arises at a temperature independent of the frequency of the probe (here at $T_{t}=75 \mathrm{~K}$ ). These effects give the transition a first-order character. It can be interpreted as due to an ordering of the dipoles and of the quadrupoles, resulting in a lower symmetry. This structural change from a cubic symmetry at high temperature to a probably tetragonal symmetry at low temperatures has been inferred from other results of Raman scattering [5], X-ray scattering [6] and birefringence measurements [7, 8] performed on samples with $\mathrm{Li}^{+}$concentrations close to that of this sample. In the sample with a lower concentration $(x=0.015)$, the behaviour, shown in figure 1 , is very different. No feature characteristic of a possible phase transition was seen in the dielectric experiments. By contrast a smooth change in the elastic constant (here near $35 \mathrm{~K}$ ) can be attributed to a transition. Thus the different nature of the low temperature phase for samples with $x>0.025$ as compared to samples with $x<0.25$ is clearly put in evidence. These results are in favour of a freezing of the $\mathrm{Li}^{+}$ions (possibly of the quadrupoles only) in these low $\mathrm{x}$ samples as suggested previously from other measurements [7]. They are called orientational glasses. Indication of the existence of a transition towards a glassy state has been confirmed by experiments with an electric field applied during cooling.

\section{RELAXATION OF THE $\mathrm{Li}^{+}$IONS.}

The second feature which is clearly seen on the dielectric and on the acoustic measurements in figures 1 and 2 is a peak in the imaginary part and a step in the real part, the temperature position of both being frequency dependent but above the transition temperature. This peak has been observed since a long time in dielectric experiments $[9,10]$. It is attributed to thermally activated movements of the $\mathrm{Li}^{+}$ions over barriers between adjacent positions ( $\pi / 2$ flip). Such a flip changes the dipolar moment and the quadrupolar moment. Therefore it is observed in the dielectric and in the acoustic measurements. A second peak can be observed for the most concentrated samples at higher temperature. This peak is not observed in the acoustic measurements. It is often called a $\pi$ flip, because in that case the quadrupolar moment is not changed. Up to now its microscopic origin is not clear. Movements of pairs of $\mathrm{Li}^{+}$ions have been suggested.

A crude analysis of these relaxation peaks, assuming a Debye behaviour (only one relaxation time $\tau$ ) with $\omega \tau=1$ at the maximum of the imaginary part ( $\omega$ is the pulsation of the probe) gives a barrier height near 
$1000 \mathrm{~K}$ for the first relaxation and a barrier height of $2700 \mathrm{~K}$ for the second relaxation. In fact, the results cannot be explained with only one relaxation time. It has been suggested that a log-normal distribution of the relaxation times, reflecting a gaussian distribution of the barriers heights, provides a good description of the data (dielectric and elastic). Another way to present the result of a relaxation phenomenum is the Argand or Cole-Cole plot, where the imaginary part is represented as a function of the real part [11,12]. Argand diagrams are shown in figure 3 for temperatures above the transition temperature for samples with low $x$ values. The shape of these diagrams is quite unusual: the slope is infinite on the left-hand side (high frequencies) while it is finite and temperature dependent on the right-hand side (low frequencies). We have called such a shape strophoidal.

Figure 3a.

Figure 3b.
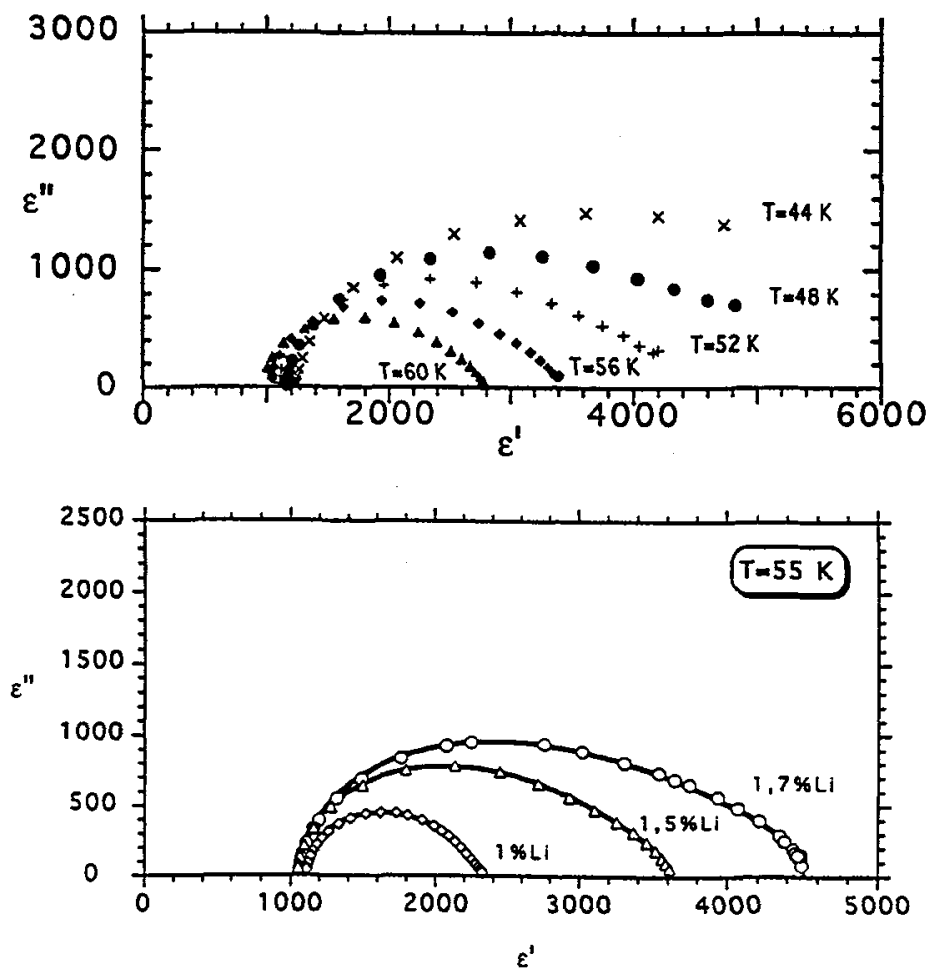

Figure $3:$ a) Argant diagrams for the $x=0.015 \mathrm{KLT}$ sample at various temperatures. b) Argand diagrams at the same temperature $\mathrm{T}=55 \mathrm{~K}$ for three different KLT crystals. The symbols represent the experimental data and the full lines the theoretical calculation.

We have shown that this strophoidal shape is related to a particular distribution of the relaxation times [11]. One of the main features of this distribution is its behaviour for large times as $\tau^{-(1+\beta)}$, with $\beta<1$. It means that the distribution function has no finite moments, in particular no mean value and no variance. It is a Lévy distribution [13]. The exponent $\beta$ is directly related to the slope on the right-hand side of the Argand diagrams which is $\beta \pi / 2$. The exponent $\beta$ increases with the temperature and decreases with the concentration $x$. These variations indicate that the correlations between $\mathrm{Li}^{+}$ions increase when $\mathrm{x}$ increases and when the temperature decreases.

The Argand diagrams plotted from the acoustic data do not permit to see the same strophoidal shape as the dielectric Argand diagrams due to the narrow frequency range attained and the less good accuracy of the data. 


\section{ERGODICITY BREAKING.}

Recently we have discovered that both the dielectric constant and the elastic constant of $\mathrm{KLT}$ crystals $[14,15]$ below their transition temperature $T_{t}$ were not equilibrium values. In other words, we have observed kinetic effects (also called aging) on these two susceptibilities over long times. A typical result for a $x=0.017$ sample is shown in figure 4 . We have tried to fit this time dependence with various laws: exponential, double exponential, stretched exponential or inverse logarithmic. We have found that our results are better described using a power law of the form $\varepsilon^{\prime}(t, \omega)=\varepsilon_{\infty}^{\prime}+A\left(\left(t+t_{0}\right) / t_{0}\right)^{-\alpha}$, where $\varepsilon_{\infty}^{\prime}, A$, to and $\alpha$ are the parameters of the fit.

The asymptotic value $\varepsilon_{\infty}^{\prime}$ appears to depend strongly on the cooling rate $R=d T / d t$ of the sample from about $100 \mathrm{~K}$ to the experiment temperature. As shown in figure 5 , faster the cooling rate is, higher the asymptotic value is. This is a non ergodic behaviour following the definition used in Classical Mechanics where a system is said ergodic when it evolves towards the same equilibrium state whatever the initial conditions are. The cooling rate dependence of $\varepsilon_{\infty}^{\prime}$ is well described by a logarithmic law as shown in figure 6 where it can be seen that the slope of $\varepsilon_{\infty}^{\prime}$ versus $\log (-\mathrm{R})$ changes with the $\mathrm{Li}^{+}$concentration. $\varepsilon_{\infty}^{\prime}$ depends on the measurement frequency at any experiment temperature in a manner which changes with the lithium concentration $x$. However, at low temperature $(4.2 \mathrm{~K})$ the kinetic part does not vary with frequency. It means that the parameters $\mathrm{A}, \mathrm{t}_{0}$ and $\alpha$ are frequency independent but they become frequency dependent at higher temperatures when the transition is approaching.

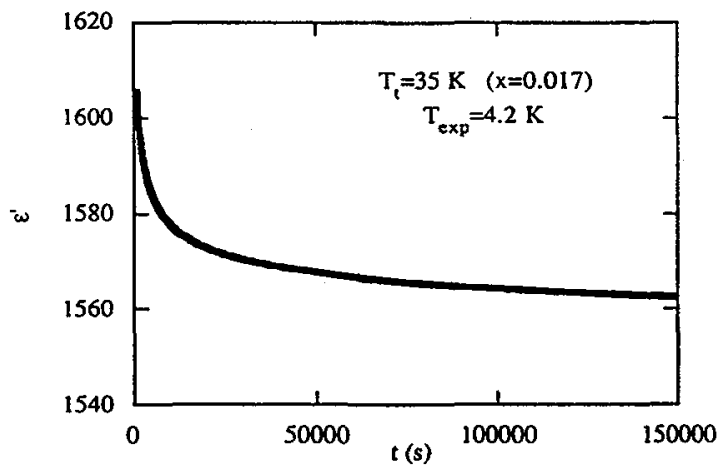

Figure 4 : Time evolution of the real part of the dielectric constant $\varepsilon^{\prime}$ measured during $150,000 \mathrm{~s}$ at the frequency $10 \mathrm{kHz}$ and the temperature $4.2 \mathrm{~K}$ for the $x=0.17$ sample.

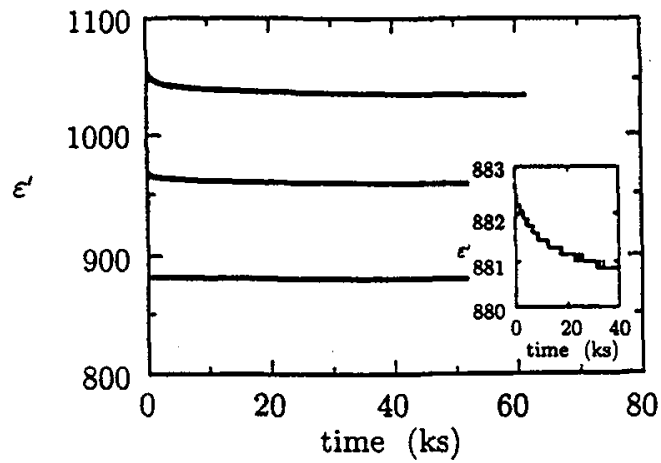

Figure 5 : Variation with time of the real part $\varepsilon^{\prime}$ of the dielectric constant of a $\mathrm{K}_{1-\mathrm{x}} \mathrm{Li}_{\mathrm{x}} \mathrm{TaO}_{3}$ sample with $\mathrm{x}=0.025$ after zero-field cooling at different rates, drawn without any shift : $R=-0.15 \mathrm{~K} / \mathrm{s}$ (upper curve) $; R=-0.02 \mathrm{~K} / \mathrm{s}$ (middle curve); $R=-0.004 \mathrm{~K} / \mathrm{s}$ (lower curve). The data were recorded at $4 \mathrm{~K}$ with an a.c. field at $100 \mathrm{kHz}$. Note that the time is measured in kiloseconds. On the vertical scale used here, the variation of the lower curve is hardly seen ; however, it is in evidence in the insert which uses an expanded vertical scale. 


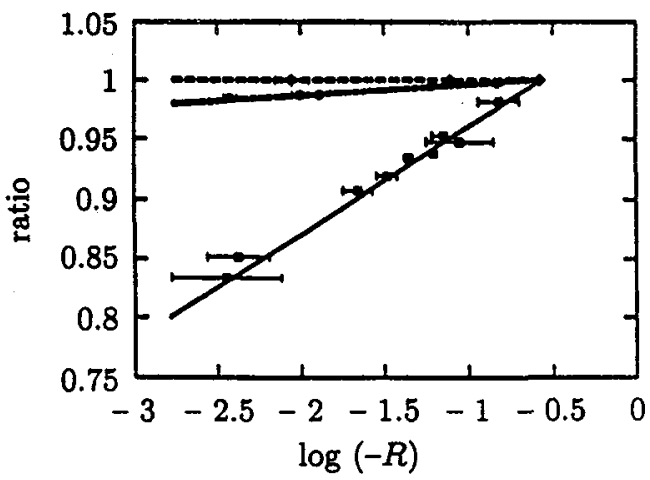

Figure 6 : Ratio of the asymptotic value $(t \rightarrow \bullet)$ of the real part of the dielectric constant divided by the asympotic value for the reference cooling rate $(R=-0.25 \mathrm{~K} / \mathrm{s}$ ), as a function of the decimal logarithm of $|R|$, for the $x=0.025$ sample (full squares), the $x=0.01$ sample (full circles) and the pure sample (full diamonds). The data were recorded at $4 \mathrm{~K}$ with an a.c. field at $100 \mathrm{kHz}$. The three lines are guides for the eye.

The elastic constant presents a similar time evolution, described by a power law. The non ergodic behaviour of the asymptotic value has also been observed.

It is worth noting that both $\varepsilon^{\prime}$ and $c^{\prime}$ decrease with time. It means that the velocity of the elastic wave decreases while the velocity of the electromagnetic wave increases.

Interesting information can be obtained from a different kind of experiments. In these cycle experiments the sample is first cooled down with a constant rate from a high temperature above $T_{1}$ to a temperature $T_{1}$ below $T_{t}$. The time evolution is first followed at $T_{1}$, then the temperature is suddenly changed to a different value $T_{2}$ also below $T_{t}$ and the time evolution is registered at $T_{2}$. Finally the temperature is another time suddenly changed back to $T_{1}$ and the time evolution is followed. The data for the two cases $T_{1}<T_{2}$ and $T_{1}>T_{2}$ are shown in figure 7 . In the first case, in each of the three time periods the system evolves towards a different asymptotic value. The time spent at the intermediate temperature $T_{2}>T_{1}$ appears only as an annealing time equivalent to a change from the initial cooling rate to a new and slower one. In the second case, $\left(T_{1}>T_{2}\right)$, the asymptotic value of the third period is the same as that of the first period. The data recorded at $T_{1}$ during the third period can be translated along the time axis in order to form an unique relaxation curve with the data recorded during the first period. The time spent at the intermediate temperature $T_{2}<T_{1}$, even if time evolution is observed during that period, has no influence on the relaxation at $T_{1}$.
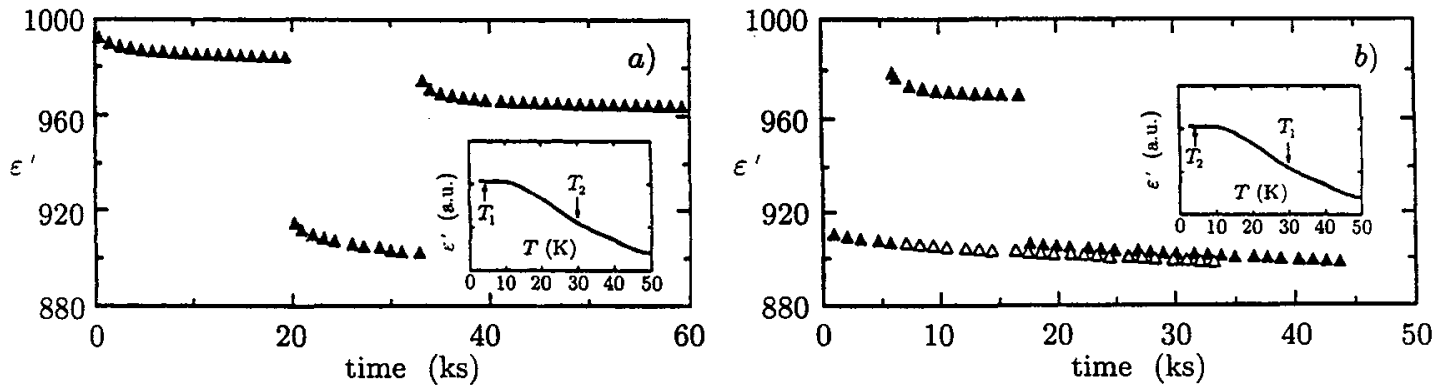

Figure 7 : Time evolution of the real part of the dielectric constant for two temperature cycles $\left(T_{1} \Rightarrow T_{2} \Rightarrow T_{1}\right)$ in the $x=0.025$ sample, measured with an a.c. field at $100 \mathrm{kHz}$. a) $T_{1}=4 \mathrm{~K}$ and $\left.T_{2}=29.5 \mathrm{~K} ; \mathrm{b}\right) \mathrm{T}_{1}=29.5 \mathrm{~K}$ and $T_{2}=4 \mathrm{~K}$. The full symbols stand for the original data, while the open symbols are used for the shifted data (see text). Only one point over ten is shown. Note that the time is measured in kiloseconds. The inserts show the temperature dependence of the dielectric constant ; the arrows mark the temperatures $\mathrm{T}_{1}$ and $\mathrm{T}_{2}$. 
These results are reminiscent of those observed in spin-glasses [16]. Aging effects have been observed in these materials but they do not show ergodicity breaking in the sense we have given here. A dependence on the thermal history of the sample have never been seen in spin-glasses. It has been said that, in spinglasses, there is a weak ergodicity breaking [17], the system is able to explore all its phase space but it needs an infinite time to do that. As a difference, in our KLT crystals, there are energy barriers of infinite heights between different regions and the system can explore only limited regions of its phase space. We can speak about our results in KLT sampies of strong or true ergodicity breaking. Moreover, in these different parts of the phase space the response to an external field is not the same (the susceptibility has different values). The behaviour of KLT crystals when submitted to temperature cycles is very similar to that of spin-glasses. In particular their relaxation measured in the second cycle $\left(T_{1}>T_{2}\right)$ reveals the same kind of hierarchical organization of the phase space in the two systems, magnetic or dielectric [18].

\section{CONCLUSION}

We want to focus on some points that we believe important. The use of two probes, (acoustic and dielectric), allows us to obtain complementary information. The different tensorial character of the two probes is a crucial point: vector (electric field) or second rank tensor (elastic strain). The two types of phase transition depending upon the $\mathrm{Li}^{+}$concentration have been confirmed: first-order character for $\mathrm{x}$ above about 0.025 and glassy character for $x$ below 0.025 . The Argand diagrams have revealed specific properties of the relaxation of the $\mathrm{Li}^{+}$especially the importance of the long times, the distribution of the relaxation times is a Lévy distribution. Kinetic effects at low temperature have been observed and studied in this orientationnal glass and a non ergodic behaviour has been clearly put in evidence.

\section{References}

[1] Höchli U. T., Knorr K. and Loidl A., Adv. Phys. 39 (1990) 405-615.

[2] Vugmeister B. E. and Glinchuk M., Rev. Mod. Phys. 62 (1990) 993-1026.

[3] Doussineau P., Farssi Y., Frénois C., Levelut A., McEnaney K., Toulouse J. and Ziolkiewicz S., Europhys. Lett. 24 (1993) 415-420.

[4] Doussineau P., Farssi Y., Frénois C., Levelut A., McEnaney K., Toulouse J. and Ziolkiewicz S., Europhys. Lett. 21 (1993) 323-328.

[5] Toulouse J., Di Antonio P., Vugmeister B. E., Wang X. M. and Knauss L. A., Phys. Rev. Lett. 68 (1992) 232-235.

[6] Andrews S. R., J. Phys. C 18 (1985) 1357-1376.

[7] Kleemann W., Kütz S. and Rytz D., Europhys. Lett. 4 (1987) 239-245.

[8] Azzini G. A., Banfi G. F., Giulotto E. and Höchli U. T., Phys. Rev. B43 (1991) 7473-7480.

[9] Höchli U. T., Phys. Rev. Lett. 48 (1982) 1494-1497.

[10] Höchli U. T. and Maglione M., J. Phys. Cond. Matter 1 (1989) 2241-2256.

[11] Doussineau P., Farssi Y., Frénois C., Levelut A., McEnaney K., Toulouse J. and Ziolkiewicz S., J. Phys. I France 4 (1994) 1217-1236.

[12] Wickenhöfer F., Kleemann W. and Rytz D. Ferroelectrics 135 (1992) 333-340

[13] Lévy P., Théorie de l'addition des variables aléatoires (Gauthier-Villars, Paris, 1954).

[14] Doussineau P., Levelut A. and Ziolkiewicz S., Europhys. Lett. 33 (1996) 539-543.

[15] Doussineau P., Levelut A. and Ziolkiewicz S., Europhys. Lett. 33 (1996) 391-396.

[16] Lefloch F., Hamman J., Ocio M. and Vincent E., Europhys. Lett. 18 (1992) 647-652.

[17] Bouchaud J.-P., J. Phys. I France 2 (1992) 1705-1713.

[18] Vincent E., Bouchaud J. P., Hamman J. and Lefloch F., Phil. Mag. 71 (1995) 489-500. 